\title{
Feature Extraction Processing Method of Medical Image Fusion Based on Neural Network Algorithm
}

\author{
Tianming Song $\mathbb{D}^{1}{ }^{1}$ Xiaoyang Yu $\mathbb{D},{ }^{1}$ Shuang $Y u,{ }^{1}$ Zhe Ren, ${ }^{1}$ and Yawei $Q u^{2}$ \\ ${ }^{1}$ The Higher Educational Key Laboratory for Measuring \& Control Technology and Instrumentations of Heilongjiang Province, \\ Harbin University of Science and Technology, Harbin 150080, China \\ ${ }^{2}$ Department of Control Science and Engineering, Harbin Institute of Technology, Harbin 150000, China
}

Correspondence should be addressed to Tianming Song; songtianming521@163.com and

Xiaoyang Yu; yuxiaoyang@hrbust.edu.cn

Received 20 August 2021; Accepted 17 September 2021; Published 8 October 2021

Academic Editor: Shaohui Wang

Copyright $\odot 2021$ Tianming Song et al. This is an open access article distributed under the Creative Commons Attribution License, which permits unrestricted use, distribution, and reproduction in any medium, provided the original work is properly cited.

\begin{abstract}
Medical image technology is becoming more and more important in the medical field. It not only provides important information about internal organs of the body for clinical analysis and medical treatment but also assists doctors in diagnosing and treating various diseases. However, in the process of medical image feature extraction, there are some problems, such as inconspicuous feature extraction and low feature preparation rate. Combined with the learning idea of convolution neural network, the image multifeature vectors are quantized in a deeper level, which makes the image features further abstract and not only makes up for the one-sidedness of single feature description but also improves the robustness of feature descriptors. This paper presents a medical image processing method based on multifeature fusion, which has high feature extraction effect on medical images of chest, lung, brain and liver, and can better express the feature relationship of medical images. Experimental results show that the accuracy of the proposed method is more than $5 \%$ higher than that of other methods, which shows that the performance of the proposed method is better.
\end{abstract}

\section{Introduction}

A wavelet neural network medical image segmentation algorithm based on the combined maximum entropy criterion is to optimize the network parameters and get the parameters so as to avoid the relative extremum and get the maximum entropy to achieve the best effect of accurate segmentation. This algorithm not only shortens the training time of samples but also greatly improves the accuracy. Compared with the traditional BP neural network, the effect is more accurate and effective [1]. With the development of medicine, medical image classification not only provides doctors with information reference but also plays a role in judging and diagnosing various diseases. Although the previous supervised convolution neural network or unsupervised CNNs methods are widely used and effective, they take a long time and cannot provide satisfactory classification accuracy [2]. Therefore, a lightweight hybrid neural network composed of improved PCANET and simplified
Densenet cascade can realize accurate classification of medical images when the training data set is small. Medical professionals usually judge diseases and make treatment plans by human abnormalities captured by imaging technology. Specialist factors, such as fatigue, rough estimates, and so on, can then limit the medical professionals' understanding of the captured images. As a tool for image understanding, convolution neural network is far superior to human experts [3]. CNN should be widely used in medical image understanding. With the wide use of computers in various fields, the assistant analysis of medical images is also an important subject. Inexperienced doctors or poorly trained radiologists can make mistakes in the diagnosis of patients' conditions. The ability to analyse and judge medical images using a data-based hierarchy would be a major step forward and prove to be the most advanced platform for achieving superior performance in a variety of biomedical applications [4]. In the aspect of medical image segmentation, the segmentation method based on CNNS is easy to 
produce unsatisfactory segmentation mask when there is no accurate target boundary. A new boundary-aware context neural network (BAC-Net) for two-dimensional medical image segmentation improves the performance of image segmentation. Generally, medical images have the characteristics of high intraclass variation and interclass blurring. The emphasis is on extracting powerful context and aggregating discriminative features for fine-grained segmentation, which can obtain richer context and retain fine spatial information [5]. Spatial attention is a weight map, and the weight map is specific.

In medicine, a group of images record the same symptom, and all images share the same weight map. Combined with this content, a new global spatial attention mechanism can be used in medical image classification [6]. It mainly uses a binary classifier to decide the boundary and takes the intensity at the pixel as the feature of the pixel. Traditional image segmentation methods for high-resolution image segmentation are difficult to achieve, which is particularly important and difficult in medical image segmentation. An image segmentation method based on optimized cellular neural network introduces nonlinear template and data quantization into the original model, which effectively reduces the computational complexity. This method is combined with the computer-aided system, combined with medical tumor X-ray photos to study tumors, and finally a multilevel optimization architecture of energy-efficient cellular neural network based on FPGA is obtained [7].

Medical image fusion can provide comprehensive and useful information to support clinical diagnosis and treatment. It uses nonsubsampling transform to divide the image into two subbands, one low frequency and one high frequency [8]. For example, in the low frequency subband, the directional feature map is extracted first, then the effective information of each directional feature map is calculated, and finally the fusion weight coefficient of the feature map guided by the function and gradient domain of image filtering is combined. The practical application of neural networks will be limited by the technical details of training these networks and the quantity and quality of manually generated annotations required. In order to fill this gap, the researchers created an interface for data annotation and neural network prediction display. It uses "people in circulation" to reduce the annotation burden. When people interact with automatically generated annotations, the segmentation of human and animal kidney microchambers has been repeatedly improved [9].

Convolution neural network (CNN) locates and retrieves medical images. It uses deep learning technology to extract powerful features of images for label description and then passes appropriate parameters to directly perform tag matching and identify them as queries [10]. Experiments show that this method is efficient. Because of the high dimension of medical image processing, the processing process is complicated. Using neural network to process medical images is as follows: (1) it shares a convolutional kernel, and high-dimensional data processing is pressure-free; and (2) there is no need to select features manually, and the weights are trained well, so that the feature classification effect is good. The feature detection layer of neural network learns by training data, so when using neural network, it avoids explicit feature extraction and learns implicitly from training data; furthermore, because the weights of neurons on the same feature mapping surface are the same, the network can learn in parallel, which is also a great advantage of convolution network compared with the network where neurons are connected with each other.

\section{Convolution Neural Network Image Processing}

As a mature means of image recognition, the learning model based on deep learning network includes convolution neural network, recurrent neural network, and important algorithms such as generating countermeasure network. In recent years, convolution neural network has achieved rapid development and broad applications and has played a huge positive role in the fields of image recognition and target detection. When using the convolution neural network model for image recognition, it is no longer necessary to design and select features manually, and the feature information needed in the recognition process is generated by $\mathrm{CNN}$ self-learning, which is constantly modified and optimized during training. In the past few decades, deep learning has proved to be a very powerful tool because of its ability to process large amounts of data. In some aspects, the performance of using deep neural network has surpassed the traditional technology, especially in pattern recognition. Convolution neural network is one of the most popular deep neural networks.

With the emergence of Image Net datasets and millions of tagged image datasets and the continuous improvement of computing power, researchers have re-realized that it is time to reuse CNN. Figure 1 is a conventional convolution neural network architecture.

Because of the diversity and complexity of the images used in the training model, the parameters will become disordered when updating the weights. If the image samples are directly input into the model, a lot of training time may be consumed, and the model is not easy to converge. In order to avoid these problems, the data input layer of convolution neural network mainly reads image samples and simple preprocessing, which provides convenience for subsequent network learning image features.

2.1. Convolution Calculation Layer. Convolution calculation layer is the most important layer in CNN structure. Convolution layer contains different numbers of convolution filters (also known as convolution kernels), which can extract different features of input image data. The operation process of convolution between image and convolution kernel can be expressed as follows:

$$
S(i, j)=(k * I)(i, j)=\sum_{m} \sum_{n} I(i+m, j+n) K(m, n),
$$




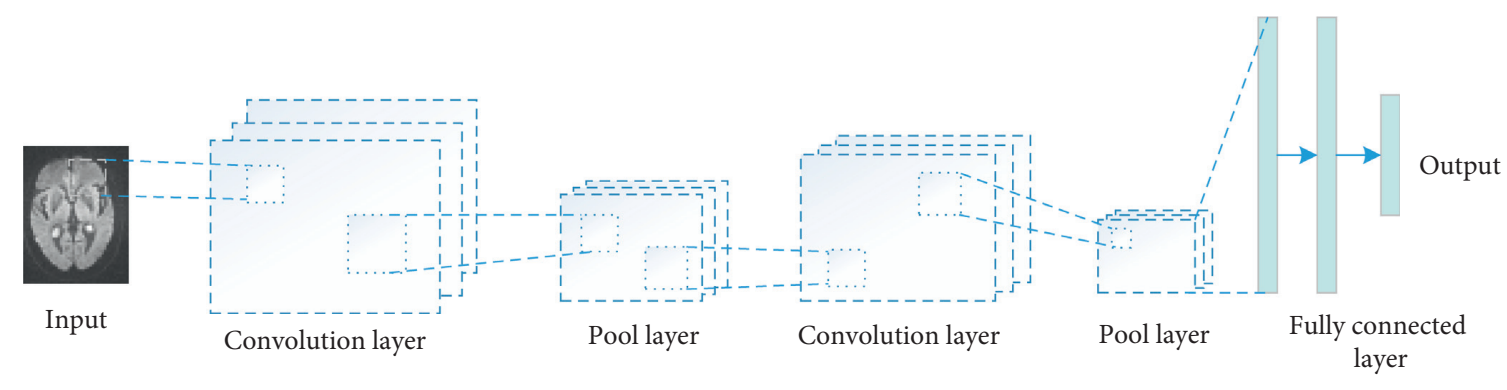

Figure 1: Convolution neural network structure of medical image.

where $I$ is the two-dimensional matrix of medical image pixels, pixels are $(m, n), S$ is the coordinates after convolution layer, which is represented by $(i, j)$, and $K$ is a convolution kernel, which can increase and decrease dimensions. The coordinates change before the image resolution changes, and the coordinates in each layer change the image resolution of each layer through convolution kernel. It is shown in Figure 2.

Convolution kernel in Figure 2 is obtained by continuously converting the image data from the sliding window, and the number of neurons determines the number of layers of the neural network. Small features are extracted from low-resolution medical images, and these small blocks are mapped to high-dimensional feature vectors. The formula for convolution operation is as follows:

$$
F_{1}(Y)=\max \left\{0, W_{1} \otimes Y+B_{1}\right\},
$$

where $Y$ represents the low-resolution input image, $\otimes$ defines the convolution operation, $W_{1}$ represents the first layer convolution operation, and the image size changes from $c \times f_{1} \times f_{2}$ size to $f_{1} \times f_{2} \times n_{1}$ matrix.

In the process of image processing, edge features are extracted, and edges are generally supplemented with 0 . It is required to keep the input and output images unchanged in dimension without changing the pixels of the original image. Figure 3 is a convolution calculation process with a step size of 2 and a convolution kernel of $3 * 3$. Its processing process is as follows.

The larger the convolution kernel is, the smoother the image will become and the less obvious the noise in the image will be. And the larger the convolution kernel is, the less obvious the contrast of the image becomes. Generally, the same dimension data convolution check results have no effect; if the dimensions of the convolution kernel are different, it will have a certain impact but other operation resources are needed.

2.2. Nonlinear Mapping. Nonlinear mapping is a process of mapping image features from one high-dimensional to another, and mapping vectors can express high-resolution features and merge these features into new feature images. In the second layer, $\mathrm{N} 1$ dimension is mapped into $\mathrm{N} 2 \mathrm{di}$ mension, which can be obtained by convolution of $f_{2} \times f_{2}$. Its formula is defined as follows:

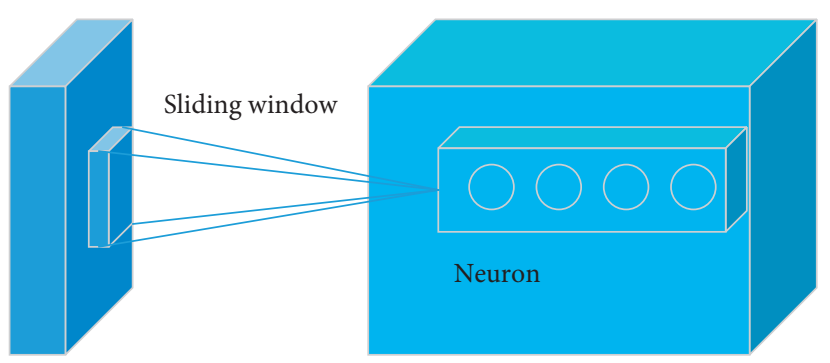

FIgURE 2: Convolution transformation diagram.

$$
F_{2}(Y)=\max \left\{0, W_{2} \otimes F_{1}(Y)+B_{2}\right\} .
$$

$W_{2}$ represents the second-level convolution operation, and its size is $f_{2} \times f_{2} \times n_{2}$, and $B 2$ is the bias of $N 2$ dimension. Through the operation of the second layer, $n_{2}$ feature-sized blocks can be obtained, as shown in Figure 4.

The final image feature can be obtained by averaging and normalizing the super eigenvalues obtained from the upper layer, as shown by the following formula:

$$
F_{3}(Y)=W_{3} \otimes F_{2}(Y)+B_{3} .
$$

2.3. Loss Function. Loss function is used to evaluate the degree to which the predicted value of the model is different from the real value. Usually, the better the loss function is used, the stronger the performance of the model is. Therefore, the loss function used by different models is generally different. Loss function is used to evaluate the fitting degree between two medical images after processing.

2.3.1. Absolute Value Loss Function. The absolute value loss function is measured by the difference between the predicted value of the algorithm and the target value, as shown by the following formula:

$$
L(Y, f(x))=|Y-f(x)|,
$$

where $Y$ represents the actual value of the image and $F(x)$ represents the predicted value of the image.

2.3.2. Logarithmic Loss Function. Object loss function is used to characterize the probability distribution of feature values in an image, especially in the case of high classification 


\begin{tabular}{|l|l|l|l|l|l|l|}
\hline 0 & 0 & 0 & 0 & 0 & 0 & 0 \\
\hline 0 & 1 & 2 & 1 & 1 & 1 & 0 \\
\hline 0 & 0 & 1 & 2 & 1 & 1 & 0 \\
\hline 0 & 1 & 0 & 2 & 1 & 0 & 0 \\
\hline 0 & 1 & 0 & 1 & 0 & 1 & 0 \\
\hline 0 & 0 & 1 & 0 & 1 & 1 & 0 \\
\hline 0 & 0 & 0 & 0 & 0 & 0 & 0 \\
\hline
\end{tabular}

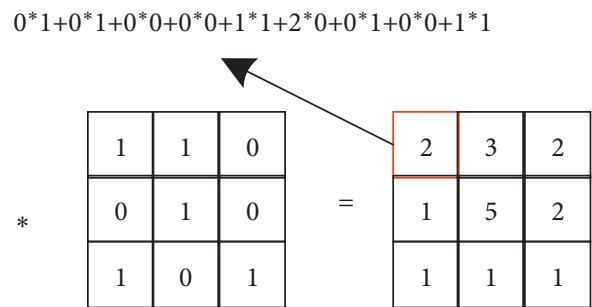

Figure 3: Convolution calculation process.

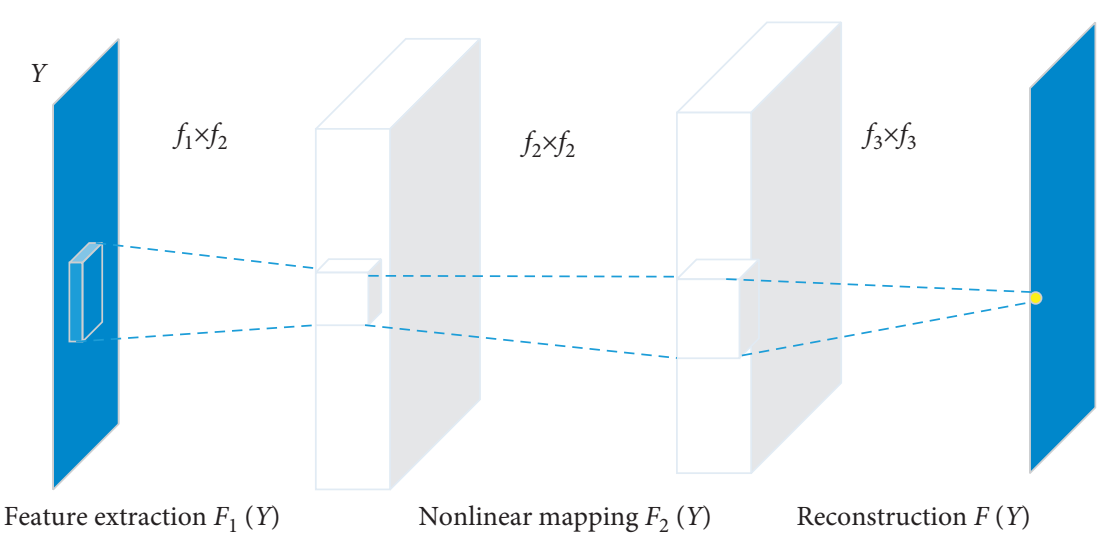

FIGURE 4: Structure diagram of nonlinear transformation neural network.

frequency. Confidence of shunting category is required. The formula is as follows:

$$
L(Y, P(Y \mid X))=-\log P(Y \mid X),
$$

where $\log P(Y \mid X)$ represents the maximum value obtained by classification $Y$ in $P(Y \mid X)$.

2.3.3. Square Loss Function. The square loss function is as follows:

$$
L(Y \mid f(X))=\sum_{n}(Y-f(X))^{2},
$$

where $Y$ is the actual value, $F(X)$ is the predicted value of the model, and $N$ is the number of samples. $Y-f(X)$ in the square loss is the residual, and the sum of squares of all residuals is the loss function value. In order to submit the practical application of the model, the variance can be used to evaluate the loss function value, and the formula is as follows:

$$
\text { MSE }=\frac{1}{N} \sum_{i=1}^{N}(P(i)-\bar{P}(i))^{2},
$$

where $P(i)$ and $\bar{P}(i)$ are actual images and reconstructed images, respectively, and $N$ is the number of samples.

2.3.4. Cross Entropy Loss Function. If $X$ is distributed in the same probability distribution $P(x)$ and $Q(x)$ for the same variable, KL is used to describe the difference between the two distributions as shown in the following formula:

$$
\begin{aligned}
D_{\mathrm{KL}}(p q) & =\sum_{i=1}^{n} p\left(x_{i}\right) \log \left(\frac{p\left(x_{i}\right)}{q\left(x_{i}\right)}\right) \\
& =\sum_{i=1}^{n} p\left(x_{i}\right) \log \left(p\left(x_{i}\right)\right)-\sum_{i=1}^{n} q\left(x_{i}\right) \log \left(q\left(x_{i}\right)\right) \\
& =-H(p(x))+\left[-\sum_{i=1}^{n} p\left(x_{i}\right) \log \left(q\left(x_{i}\right)\right)\right] .
\end{aligned}
$$

2.4. Activation Function. Convolution neural network is composed of excitation function. The activation function in this paper adopts nonlinearity to characterize complex characteristics, which is shown in the following formula:

$$
A_{i, j, k}^{l}=f\left(Z_{i, j, k}^{l}\right) .
$$

Similar to multilayer neural networks, convolution neural networks use Sigmoid functions, and common activation functions are as follows:

$$
\operatorname{ReLU}: f_{\mathrm{ks}}(x)=\max (x, 0) \text {, }
$$

Sigmoid: $f_{k s}(x)=\frac{1}{1+e^{-x}}$, 
Hyperbolic tangent: fks $(x)=\tanh (x)=\frac{e^{x}-e^{-x}}{e^{x}+e^{-x}}$.

Activate functions are used after convolution operations, and some use preactivate function algorithms before convolution.

\section{Medical Image Detection Algorithm}

The traditional medical image processing process and neural network detection algorithm show abnormal data matching. However, the traditional segmentation algorithm makes the extraction of medical image feature value to have unreasonable features. Therefore, the main key parts of the medical image detection algorithm can be described as four parts: medical image segmentation, feature region extraction, feature region analysis and extraction, and medical image feature classification and recognition.

The image area of interest to the medical image is obtained, and the image from the area with obvious characteristics of the medical image is obtained. In the realization of image processing, the threshold method is used for segmentation; then, feature segmentation and feature extraction are carried out on the region of interest; the final extracted feature values are standardized, and the neural network is used to detect and identify the image. The detection process is shown in Figure 5.

3.1. Standardization of Medical Images. In the neural network, because the number of layers is more, with the increasing scale of the network, the data value is constantly shifted, which will lead to the optimization of the data value and produce huge errors. Therefore, the data in different layers of the convolution network are optimized, and the deviation of input data caused by different layers is reduced. In this paper, batch gradient descent is used to optimize the convolution neural network, and grouping is used to update the data. At each layer of the neural network, the input is defined as $X=\left[x_{1}, x_{2} \ldots x_{n}\right], X_{i}$ is a group of samples, and $N$ is the number of groups. The standardization process is as follows:

Step 1.Take mean of each group of elements:

$$
\mu=\frac{1}{n} \sum_{i=1}^{n} x_{i}
$$

Step 2. Find the variance again:

$$
\sigma^{2}=\frac{1}{n} \sum_{i=1}^{n}\left(x_{i}-\mu\right)
$$

Step 3. Normalize each pixel again:

$$
x_{i}^{\prime} \frac{x_{i}-\mu}{\sqrt{\sigma^{2}+\varepsilon}}
$$

Step 4. The final output is $y_{i}$ based on the offset:

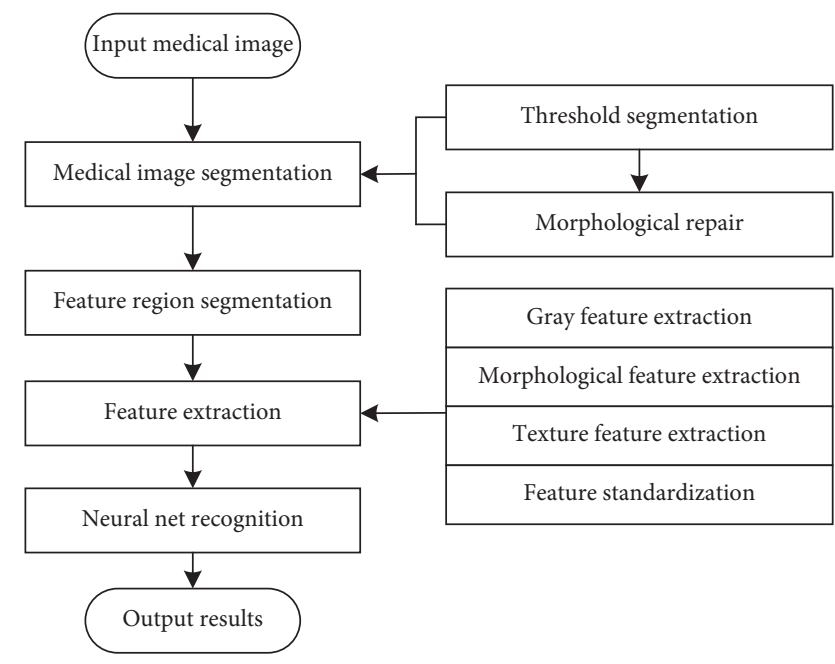

Figure 5: Medical image feature extraction and recognition process.

$$
y_{i}=\gamma_{i} * x_{i}+\beta
$$

3.2. Medical Image Segmentation Method. The threshold method is a segmentation method based on attributes, such as gray features and image pixels. Its expression is as follows:

$$
G(x, y)= \begin{cases}0, & f(x, y)<T \\ 255, & f(x, y)>T .\end{cases}
$$

Formula (18) is a single threshold segmentation, and the segmented image is not ideal under complex image information. The global threshold method is an adaptive segmentation method based on the different features of the image. The process is as follows:

Step 1. In the initialization process, select an optimal estimated value as $T$ of the global threshold and generally select the average value of the maximum and minimum, as shown in the following formula:

$$
T=0.5(\min G(:)+\max G(:)) \text {. }
$$

Step 2. According to the threshold $T$, segment the image into two groups, respectively, $G 1$ and $G 2$, and the gray value is larger $T$ in $G 1$, and the gray value is less than $T$ in G2;

Step 3. Calculate the average gray value, and calculate the average value of $G 1$ and $G 2$ image sets, respectively, that is $m_{1}$ and $m_{2}$.

Step 4. Update the $T$ value as shown in the following equation:

$$
T=\frac{1}{2}\left(m_{1}+m_{2}\right) .
$$

Repeat Step 1 through Step 4 until the Tvalue is less than the specified value. 


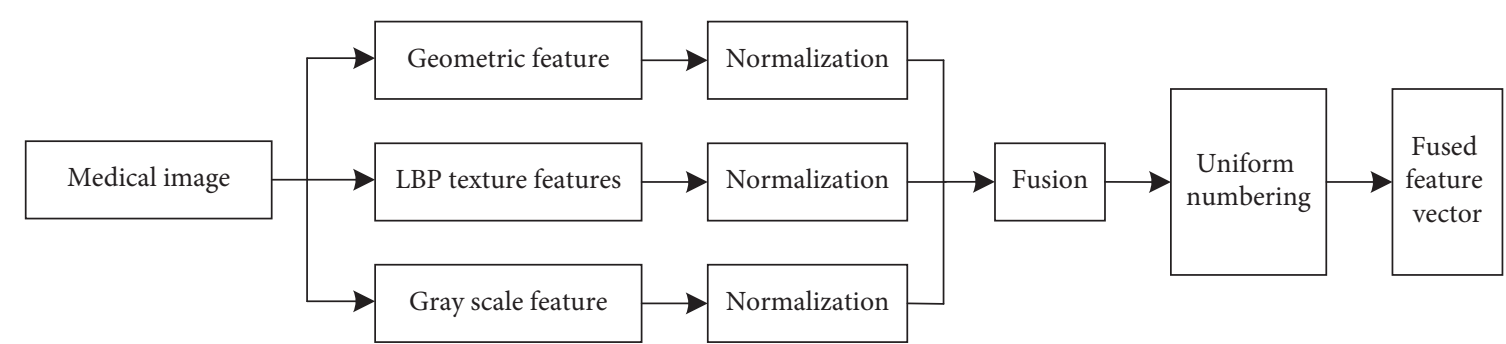

FIgURE 6: Feature fusion process.

TABLE 1: Experimental environment settings.

\begin{tabular}{lc}
\hline Environment configuration & Model \\
\hline GPU & NVIDIA RTX3090 24G \\
CPU & Intel silver 4210R 10 cores 20 threads \\
Memory & $64 \mathrm{G}$ \\
Operating system & Ubuntu 16.04 \\
Language & Python 3.7 \\
\hline
\end{tabular}

\subsection{Feature Analysis and Extraction of Medical Images}

3.3.1. Gray Scale Characteristics. The gray features of medical images are obvious Peugeot features, including gray mean, gray variance, and gray histogram entropy. They are shown in formulas (21)-(23), respectively.

$$
\begin{aligned}
G_{m} & =\frac{\sum_{i=0}^{M} \sum_{j=0}^{N} I(i, j)}{M \times N}, \\
G_{n} & =\sum_{i=0}^{M} \sum_{j=0}^{N}\left(I(i, j)-G_{m}\right)^{2}, \\
H & =-\sum_{i=0}^{L-1} p(i) \log _{2} p(i) .
\end{aligned}
$$

3.3.2. Texture Features. Texture feature is a part of the image statistics and is based on the image of multiple pixels of the features, and in medical images, the lesion part of the features is more obvious, and some features also have interference. Texture features can be represented by gray-level cooccurrence matrix, which can not only express interesting features in medical images. The gray-level co-occurrence matrix can be described by the following formula:

$$
\mathrm{ASM}=\sum I(i, j)^{2}
$$

where $I(i, j)$ refers to the standard gray-level co-occurrence matrix.

$$
\begin{aligned}
& \mathrm{ENT}=\sum I(i, j)(-\ln (I(i, j))), \\
& \mathrm{IDM}=\sum \frac{I(i, j)}{1+(i-j)^{2}} .
\end{aligned}
$$

The local binary model is used to calculate the texture features of the image, which can better express the gray values of adjacent areas, and the center values of each area are solved by weighting each. The formula is as follows:

$$
\left\{\begin{array}{c}
\operatorname{LBP}\left(x_{c}, y_{c}\right)=\sum_{i=0}^{n-1} s\left(f_{i}, f_{c}\right) 2^{i}, \\
s\left(f_{i}, f_{c}\right)= \begin{cases}1, & f_{i}>f_{c}, \\
0, & f_{i}<f_{c},\end{cases}
\end{array}\right.
$$

where $\left(x_{c}, y_{c}\right)$ is the center pixel, $f_{c}$ is the center pixel value, $f_{i}$ is the neighbor gray value, and $n$ is the number of leading neighbors.

3.3.3. Feature Fusion. Image Shape Feature Pair for image approximates shape and appearance features; multifeature fusion is used to enhance the feature region of the image to improve the accuracy of image feature extraction. Multifeature fusion is the fusion of different feature extraction results to characterize the image content so as to accurately express the image information. The process is shown in Figure 6.

However, these three feature fusion methods have some requirements in feature dimensions, such as the features participating in the fusion should have the same feature dimensions. The normalization mentioned here is the standardization process mentioned earlier, which improves the accuracy of data fusion.

\section{Experimental Comparison and Analysis}

4.1. Experimental Preparation. In the convolution neural network used in this paper, the activation function is Sigmoid function and the loss function is cross entropy loss function. The experiment needs a better experimental hardware environment, and the experimental environment adopted in this paper is shown in Table 1.

The Keras deep learning model is used to establish a network model and a convolution neural network fused with features in Figure 7.

From the above results, it can be seen that the image processing method based on fused image features has obvious segmentation effect. After processing the images that are not key features, the obvious parts of features are displayed. 


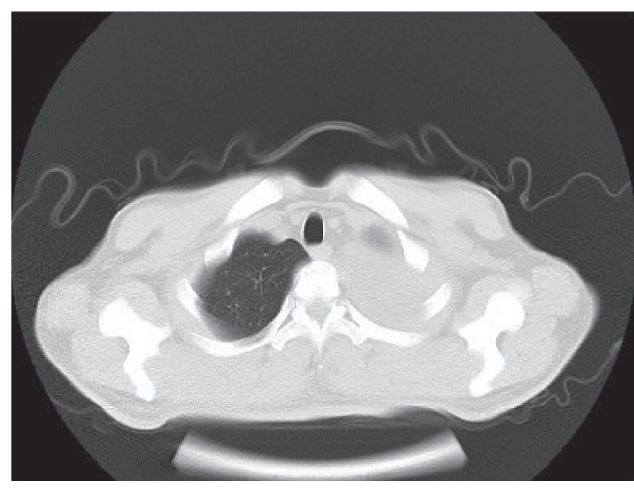

(a)

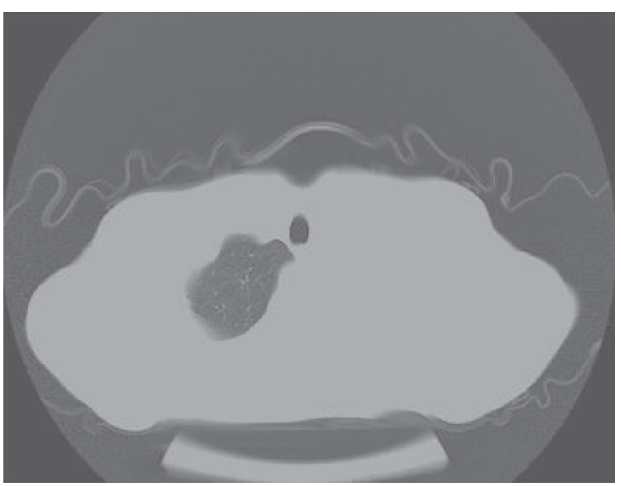

(c)

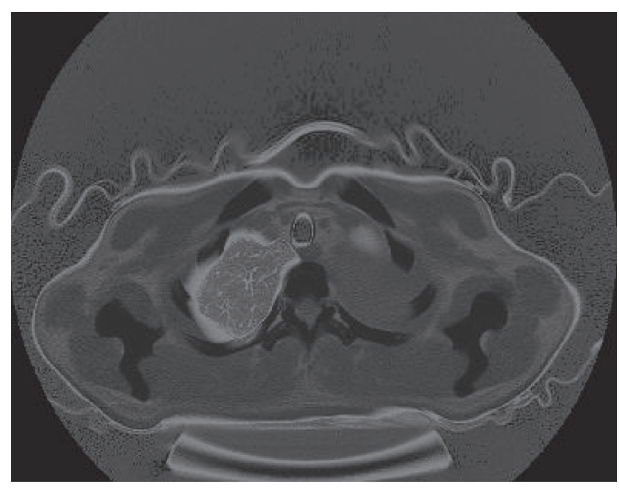

(b)

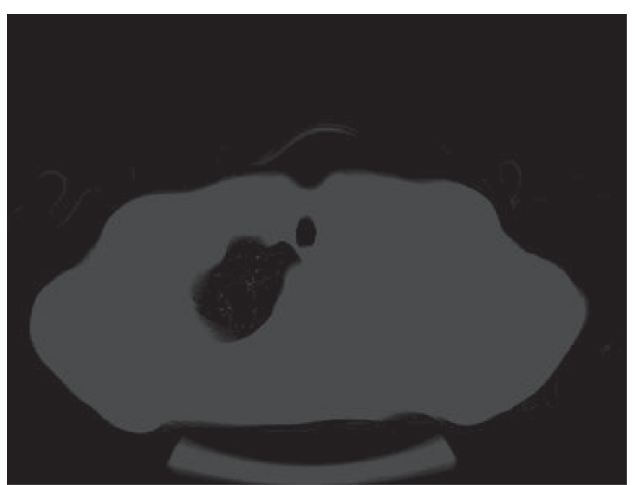

(d)

FIGURE 7: Comparison of algorithms under different feature images: (a) original image; (b) SURF; (c) gray texture feature; (d) fusion feature method.

4.2. Comparison of Accuracy of Different Medical Images. Different numbers of return results $K(8,16,24$, and 32$)$ are set for images of different parts of the human body, and the precision of the images is counted. It can be seen from Table 2 that the precision is obviously improved after fusing SURF corner features and gray-level symbiotic texture features. When $K=8$, the precision rate is over $80 \%$, which shows that the similarity of the results is very high in the image processing process.

There are medical images of different parts in the data set. According to the above method, the images of chest, lung, brain, and liver are fused with features. Twenty medical images from each part of the data set are randomly selected as feature tests, and the average precision is calculated by returning $K$ feature extraction results, where $K$ value is 24 . Table 3 shows the experimental results of the average precision of medical images in different parts under three characteristics.

Table 3 shows the retrieval precision of medical images such as chest, lung, brain, and liver under three feature extraction algorithms. Analysis shows that the four types of the image retrieval algorithm in this chapter compared with a single feature retrieval precision have significantly improved. It shows that it is very necessary to integrate texture features into medical image retrieval to make up for the defects of single feature in medical image content description.
TABLE 2: Comparison of precision rates of several feature extraction methods.

\begin{tabular}{lccc}
\hline$K$ & SURF & Gray texture feature & Fusion feature \\
\hline 8 & 76.83 & 64.34 & 84.35 \\
16 & 65.24 & 68.63 & 75.12 \\
24 & 55.95 & 67.25 & 74.36 \\
32 & 54.21 & 64.35 & 75.52 \\
\hline
\end{tabular}

This paper verifies the linear coding method of fusion angle points and texture features to improve the retrieval accuracy. The algorithm in this chapter is compared with SURF, gray texture feature extraction, and Gabor texture feature extraction. The comparison of experimental results is shown in Figures 8-11.

Figures $8-11$ show the retrieval precision of medical images of chest, lung, brain, and liver under four feature extraction algorithms. Analysis shows that the four types of the image retrieval algorithm in this chapter compared with a single feature retrieval precision have significantly improved. It shows that it is very necessary to incorporate texture features in medical image feature extraction, and the feature fusion method proposed in this paper has a good performance in feature extraction accuracy of different parts of the human body, which is about $5 \%$ higher in specific medical image types. 
TABLE 3: Comparison of feature precision rates of medical images in different parts.

\begin{tabular}{lccc}
\hline Image type & SURF & Gray texture feature & Fusion feature \\
\hline Chest & 73.41 & 68.25 & 81.47 \\
Lung & 70.21 & 72.65 & 79.35 \\
Brain & 68.54 & 70.25 & 75.24 \\
Liver & 65.24 & 65.21 & 71.65 \\
\hline
\end{tabular}

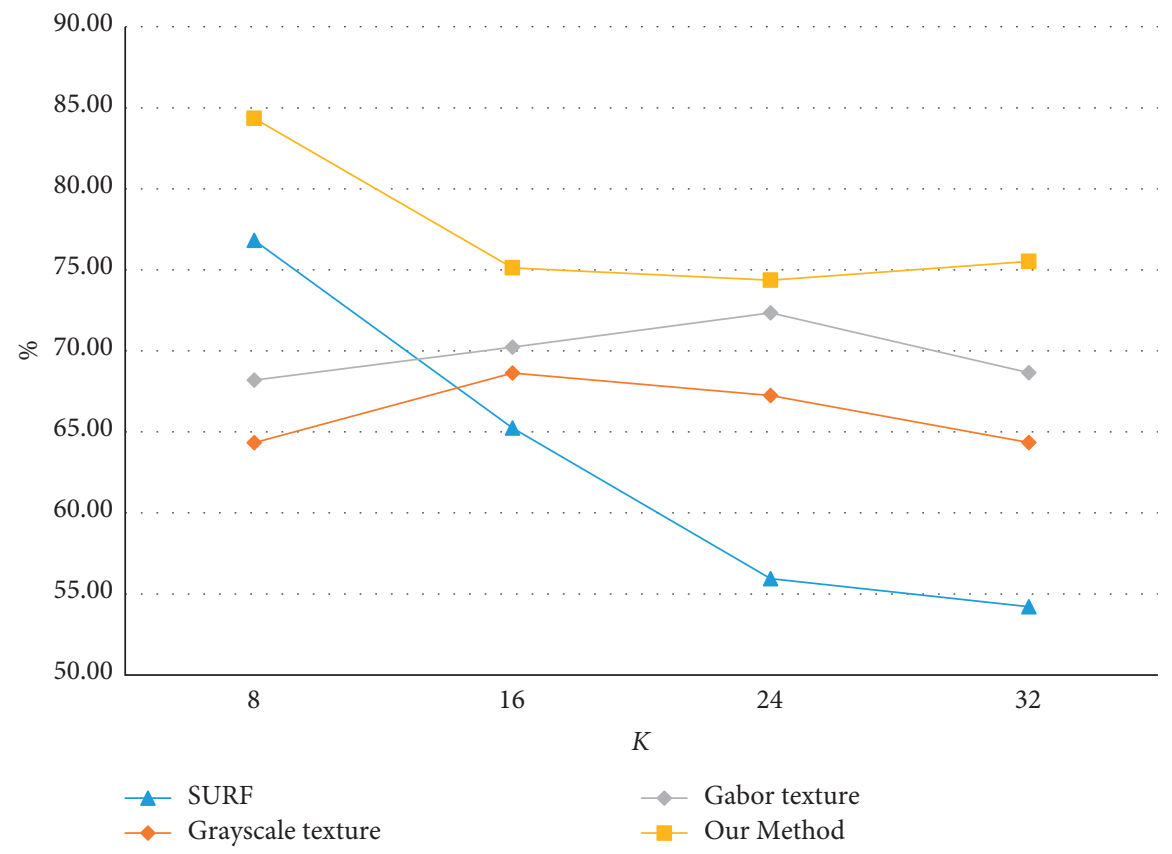

Figure 8: Comparison of features of chest medical images.

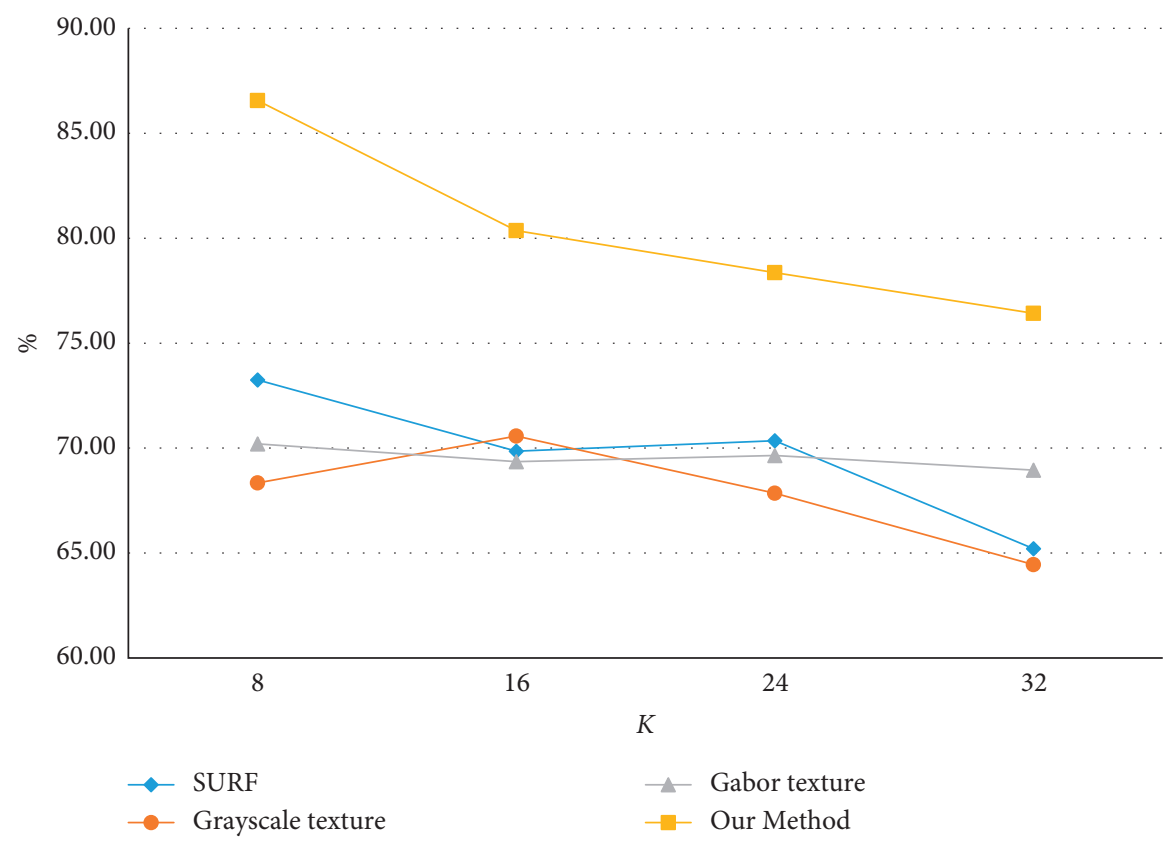

FIgURE 9: Comparison of features of lung medical images. 


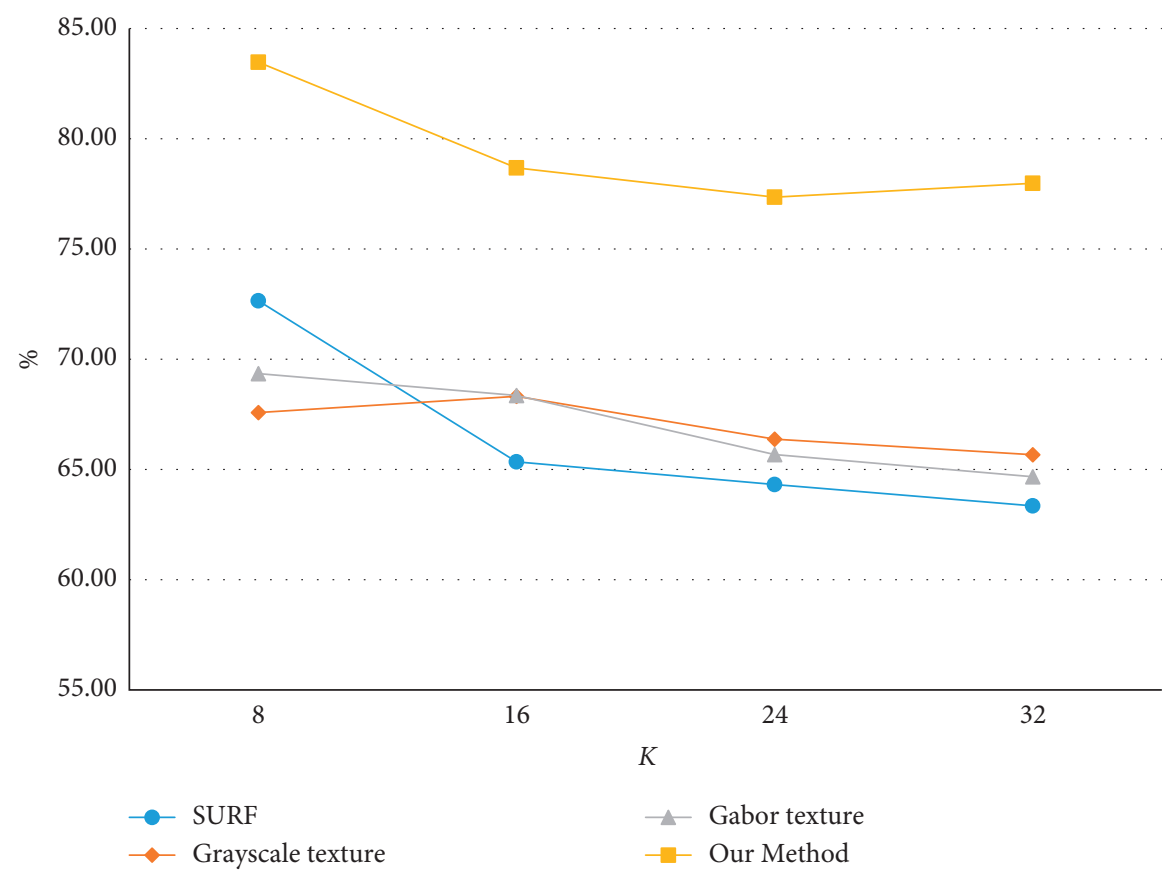

Figure 10: Comparison of features of brain medical images.

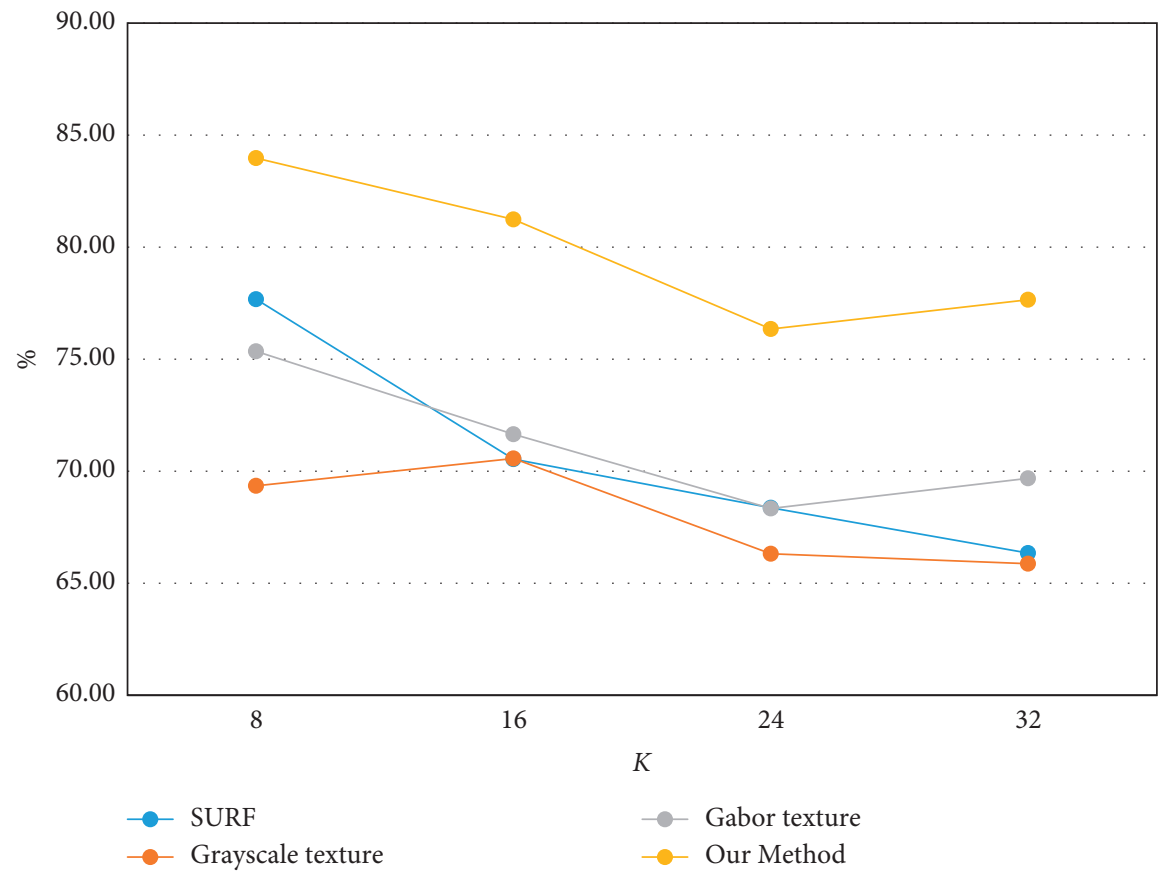

Figure 11: Comparison of features of liver medical images.

\section{Conclusion}

This paper presents a medical image processing method based on fusion of multiple features, which has high feature extraction effect on medical images of chest, lung, brain, and liver, and can better express the feature relationship of medical images. Feature extraction is very important in medical image processing. The features can be extracted effectively from the fusion of multifeatures, which shows the correctness and applicability of the method proposed in this paper. This paper compares four kinds of medical images, and there are still many medical images that have not been studied and compared. In the later stage, convolution neural network will be introduced to fuse multifeature images for feature extraction. 


\section{Data Availability}

The experimental data used to support the findings of this study are available from the corresponding author upon request.

\section{Conflicts of Interest}

The authors declare that they have no conflicts of interest regarding this work.

\section{Acknowledgments}

This work was supported in part by Heilongjiang Postdoctoral Financial Assistance (LBH-Z19071).

\section{References}

[1] Z. Huang, X. Zhu, M. Ding, and X. Zhang, "Medical image classification using a light-weighted hybrid neural network based on PCANet and DenseNet," IEEE Access, vol. 8, no. 99, pp. 24697-24712, 2020.

[2] X. Wu, H. Chen, X. Wu, S. Wu, and J. Huang, "Burn image recognition of medical images based on deep learning: from CNNs to advanced networks," Neural Processing Letters, vol. 53, no. 1, pp. 1-18, 2021.

[3] D. R. Sarvamangala and R. V. Kulkarni, "Convolutional neural networks in medical image understanding: a survey," Evolutionary Intelligence, no. 4, pp. 1-22, 2021.

[4] S. Verma and R. Agrawal, "Deep neural network in medical image processing," Handbook of Deep Learning in Biomedical Engineering, Academic Press, Cambridge, MA, USA, pp. 271-292, 2021.

[5] Z. Gu, J. Cheng, H. Fu et al., "CE-net: context encoder network for 2D medical image segmentation," IEEE Transactions on Medical Imaging, vol. PP, no. 99, p. 1, 2019.

[6] Y. Liang, H. Li, B. Guo et al., "Fusion of heterogeneous attention mechanisms in multi-view convolutional neural network for text classification," Information Sciences, vol. 548, no. 2021, pp. 295-312, 2020.

[7] X. Wang, L. Gu, and Z. Wang, "Computer medical image segmentation based on neural network," IEEE Access, vol. PP, no. 99, p. 1, 2020.

[8] G. Wang and Y. Huang, "Medical image fusion based on modified pulse coupled neural network model and kirsch operator," International Journal of Wavelets, Multiresolution and Information Processing, vol. 17, no. 1983, p. 6, 2019.

[9] B. Lutnick, B. Ginley, D. Govind et al., "An integrated iterative annotation technique for easing neural network training in medical image analysis," Nature Machine Intelligence, vol. 1, no. 2, pp. 112-119, 2019.

[10] N. Kalaivani, D. Devi, D. S. Sophia, N. Manimaran, and P. Kowsalya, "Medical image retrieval using convolution neural networks," IOP Conference Series: Materials Science and Engineering, vol. 994, Article ID 012038, 2020. 